\title{
Association of Albumin to Creatinine Ratio with Cardiovascular Risk Markers and Determination of Their Cut off Points in Type 2 Diabetic Nephropathy Patients
}

\author{
Bhoite $\mathrm{GM}^{1}$, Bulakh $\mathrm{PM}^{2}$, Kuvalekar $\mathrm{AA}^{3}$, Momin $\mathrm{AA}^{2}$ \\ ${ }^{I}$ (Department Of Human Physiology \& Biochemistry, BVDU Dental College, Pune, India) \\ ${ }^{2}$ (Department Of Biochemistry, BVDU Medical College, Pune, India) \\ ${ }_{3}^{3}$ Interactive Research School For Health Affairs, Bharati Vidyapeeth Deemed University, Pune, India)
}

\begin{abstract}
Urine albumin to creatinine ratio (ACR) is found to be the most promising indicator for confirmation of nephropathy in type 2 diabetes mellitus patients. It is also known that the increased excretion of urine microalbumin is associated with high risk of cardiovascular diseases. The present study was aimed to attain the association of BMI, FBG, HbAlc, cardiovascular markers, urine creatinine \& urine microalbumin, and also to find out the cut off points of these markers for the prediction of cardiovascular disease in type 2 diabetic nephropathy patients. We included 110 type 2 diabetic nephropathy patients and 110 healthy age and gender matched healthy controls. The value of BMI, and levels of FBG, triglycerides, VLDL cholesterol, urine creatinine and urine microalbumin were found to be positively associated and HDL cholesterol was negatively associated with ACR in type 2 diabetic nephropathy patients. Further, ROC curve analysis of BMI, total cholesterol, triglycerides, HDL cholesterol, LDL cholesterol and VLDL cholesterol gave the cut off values of $>24.3,>187.2,>143.1, \leq 42.7,>119.9$ and $>37.2$, respectively with significant area under the ROC curve, highest sensitivity and highest specificity. From these results we conclude that the ACR apart from its function as an indicator of nephropathy can also serve as an indicator of cardiovascular risk in type 2 diabetic patients. These cut off values can be used for prediction of cardiovascular disease risk in type 2 diabetic nephropathy patients.
\end{abstract}

Keywords: Albumin to creatinine ratio, cardiovascular disease, triglycerides (TGs), type 2 diabetic nephropathy

\section{Introduction}

The incidence of diabetes mellitus is increasing globally, with its potential complications like heart disease, stroke, and kidney damage. [1] Diabetes is characterized by hyperglycemia, in most cases of diabetes; occurrence of hypertension is seen sooner or later, which is an established causal factor for diabetic nephropathy (DN). [2] DN is one of the most common clinical conditions leading to end stage renal disease (ESRD). [3] It is a life threatening complication resulting in a poor prognosis for patients as well as high medical costs. [4] The pathophysiological mechanisms of DN are attributed primarily to metabolic and hemodynamic derangements including hyperglycemia induced production of advanced glycation end products (AGEs), activation of polyol pathway, protein kinase $C$ and rennin angiotensin system. $[5,6]$ The earliest demonstrable abnormalities include intrarenal hypertension, increased glomerular filtration rate, and microalbuminuria. [7] The characteristic increased albumin excretion of nephropathy, even microalbuminuria, is known to increase the risk of cardiovascular disease in diabetic as well as non-diabetic patients. The mechanism of this may be linked to the increased levels of atherogenic lipoproteins promoted by endothelial dysfunction. [8, 9] Albuminuria ( $>300 \mathrm{mg} / 24$ hours) or microalbuminuria ( $\geq 30 \mathrm{mg} / 24$ hours to $300 \mathrm{mg} / 24$ hours), or albumin/creatinine ratio ( $\geq 30$ $\mathrm{mg} / \mathrm{g}$ ), is used as a marker of renal damage and is used to define chronic kidney disease along with low estimated glomerular filtration rate (eGFR). [10]

Both cardiovascular disease and diabetes mellitus share many risk factors in common (the "common soil" hypothesis), [11] also lipid metabolism has been extensively investigated in diabetes; little information is available whether urine ACR is associated with lipids and lipoproteins. Therefore, we hypothesized that the cardiovascular markers are associated with ACR, cut off values of lipids and lipoproteins can be calculated for the prediction of cardiovascular disease.

\section{Material And Methods}

The present study consisted of, 110 T2DM patients with nephropathy within the age limit of 35-60 years. Patients visiting Medicine Department, BVDU Medical College and Hospital, Pune were included in the study. T2DM nephropathy patients, having cardiovascular, pulmonary diseases, with diabetic complications other than nephropathy, pregnant and lactating women and those on insulin therapy were excluded from the 
study. In addition to T2DM nephropathy patients, age and gender matched 110 healthy non-diabetic individuals were also included in the study. The healthy individuals had no history of cardiovascular disorders or any major illness. The research protocol was accepted by Institutional Ethical Committee of BVDU Medical College, Pune (BVDU/MC/55/2013-2014) and informed written consent was obtained from every individual before any study related activity.

The blood samples were collected by venipuncture after fasting of 12 hours. Plasma from the blood collected in fluoride vacutainer was used for estimation of fasting blood glucose (FBG) using commercially available kit (ERBA Diagnostics, Mannheim, Germany), and glycated hemoglobin (HbA1c) was estimated from samples collected in EDTA vacutainer by HPLC technique (D10, Bio-Rad). The serum was separated from plain vacutainer after centrifugation, and used for estimation of total cholesterol (TC), TGs and high density lipoprotein cholesterol (HDL cholesterol). Low Density Lipoprotein cholesterol (LDL cholesterol) and Very Low Density Lipoprotein cholesterol (VLDL cholesterol) were calculated by Friedwald's formula. Urine samples collected were used for estimation of urine creatinine and microalbumin level, and from these values the ratio of ACR was calculated. All biochemical parameters were estimated by standard, commercially available kits (ERBA Diagnostics, Mannheim, Germany).

The correlation between ACR and cardiovascular markers was tested by Pearson's correlation coefficient. Further, the cut off values of cardiovascular markers were calculated at highest sensitivity and specificity with corresponding area under the curve by Receiver Operating Characteristics (ROC) curve analysis. The $\mathrm{P}$ values $\leq 0.05$ were considered significant.

\section{Results}

The ACR was calculated from urine albumin and urine creatinine values, then the correlation of ACR with BMI, FBG, HbA1c, cardiovascular markers, urine albumin and creatinine was evaluated. The relevant details of association of ACR with BMI, FBG, HbA1c, cardiovascular markers, urine microalbumin, and urine creatinine are represented in table 1 and figure $1 \mathrm{a}$ to $1 \mathrm{~g}$ shows respective scatter diagram. ' $\mathrm{r}$ ' denotes correlation coefficient with $95 \%$ confidence interval and respective ' $p$ ' values..

Table 1: Association of ACR with biochemical parameters in type 2 diabetic nephropathy patients

\begin{tabular}{|l|c|c|c|}
\hline & $\mathbf{r}$ & $\mathbf{9 5 \%} \mathbf{C I}$ & $\mathbf{P}$ \\
\hline BMI & 0.5030 & 0.3486 to 0.6309 & $<0.0001^{*}$ \\
\hline FBG & 0.2525 & 0.06845 to 0.4199 & $0.0078^{*}$ \\
\hline HbA1c & 0.1040 & -0.08492 to 0.2857 & 0.2797 \\
\hline Total Cholesterol & 0.1005 & -0.08843 to 0.2824 & 0.2963 \\
\hline Triglycerides & 0.2859 & 0.1043 to 0.4491 & $0.0025^{*}$ \\
\hline HDL-C & -0.3213 & -0.4797 to -0.1426 & $0.0006^{*}$ \\
\hline LDL-C & 0.07630 & -0.1126 to 0.2598 & 0.4282 \\
\hline VLDL-C & 0.2861 & 0.1044 to 0.4493 & $0.0024^{*}$ \\
\hline Urine creatinine & 0.2990 & 0.1184 to 0.4605 & $0.0015^{*}$ \\
\hline Urine microalbumin & 0.6986 & 0.5884 to 0.7834 & $<0.0001^{*}$ \\
\hline
\end{tabular}

* Statistically significant, r: Correlation coefficient; BMI: Body mass index; FBG: Fasting blood glucose; HDL-C: High density lipoprotein cholesterol; LDL-C: Low density lipoprotein cholesterol; VLDL-C: Very low density lipoprotein cholesterol; CI: Confidence Interval
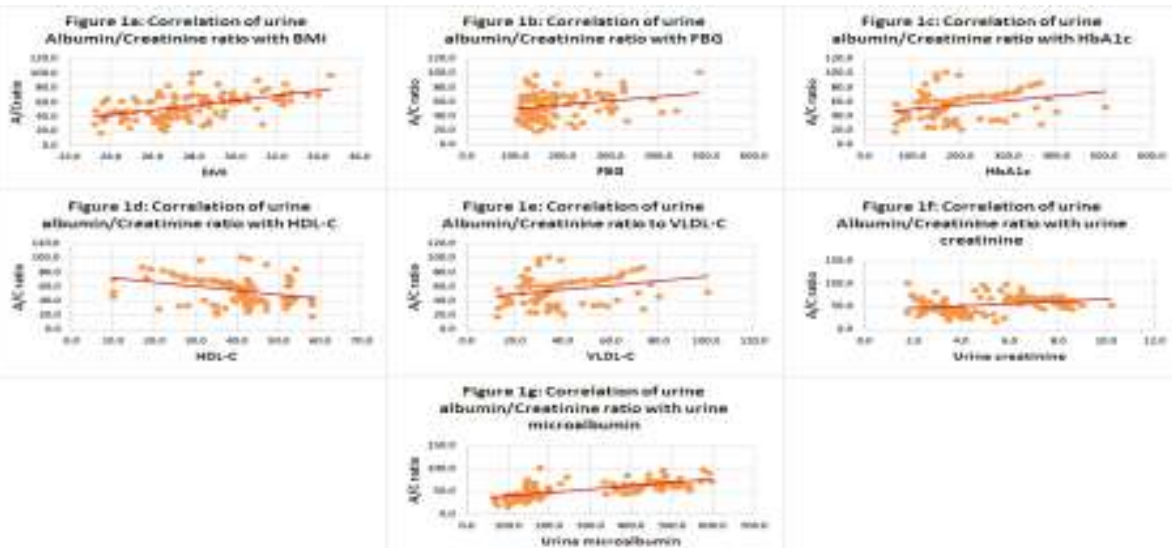

Figure 1: The scatter diagrams of biochemical parameters in patients with type 2 diabetic nephropathy 
It was observed that BMI $(\mathrm{p}<0.0001)$, FBG $(\mathrm{p}=0.0078)$, TG $(\mathrm{p}=0.0025)$, VLDL-C $(\mathrm{p}=0.0024)$, urine creatinine $(\mathrm{p}=0.0015)$ and urine microalbumin $(\mathrm{p}<0.0001)$ were positively associated while HDL-C $(\mathrm{p}=0.0006)$ was negatively associated with ACR.

Table 2: The area under the ROC curves with $95 \%$ confidence interval of BMI and cardiovascular markers for type 2 diabetic nephropathy patients

\begin{tabular}{|l|c|c|c|}
\hline \multicolumn{1}{|c|}{ Parameters } & AUC \pm SE & 95\% CI & p value \\
\hline BMI & $0.825 \pm 0.0275$ & 0.768 to 0.873 & $<0.0001^{*}$ \\
\hline Total cholesterol & $0.724 \pm 0.0342$ & 0.660 to 0.782 & $<0.0001^{*}$ \\
\hline Triglycerides & $0.725 \pm 0.0341$ & 0.661 to 0.783 & $<0.0001^{*}$ \\
\hline HDL-C & $0.823 \pm 0.0322$ & 0.766 to 0.871 & $<0.0001^{*}$ \\
\hline LDL-C & $0.730 \pm 0.0338$ & 0.667 to 0.788 & $<0.0001^{*}$ \\
\hline VLDL-C & $0.725 \pm 0.0341$ & 0.661 to 0.783 & $<0.0001^{*}$ \\
\hline
\end{tabular}

* Statistically significant, AUC: Area under the ROC curve; HDL-C: High density lipoprotein cholesterol; LDL-C: Low density lipoprotein cholesterol; VLDL-C: Very low density lipoprotein cholesterol; SE: Standard Error; CI: Confidence Interval

Table 3: The cut off points for BMI and cardiovascular markers with corresponding highest \%sensitivity and $\%$ specificity

\begin{tabular}{|l|c|c|c|c|c|}
\hline & Cut off value & Specificity $(\boldsymbol{\%})$ & $\mathbf{9 5 \%} \mathbf{C I}$ & $\begin{array}{c}\text { Sensitivity } \\
(\boldsymbol{\%})\end{array}$ & $\mathbf{9 5 \%}$ CI \\
\hline BMI & $>24.3$ & 90.91 & $83.9-95.6$ & 54.55 & $44.8-64.1$ \\
\hline $\begin{array}{l}\text { Total } \\
\text { Cholesterol }\end{array}$ & $>187.2$ & 60.00 & $50.2-69.2$ & 74.55 & $65.4-82.4$ \\
\hline Triglycerides & $>143.1$ & 71.82 & $62.4-80.0$ & 58.18 & $48.4-67.5$ \\
\hline HDL-C & $\leq 42.7$ & 71.82 & $62.4-80.0$ & 93.64 & $87.3-97.4$ \\
\hline LDL-C & $>119.9$ & 70.91 & $57.1-82.4$ & 86.36 & $78.5-92.2$ \\
\hline VLDL-C & $>37.2$ & 58.18 & $44.1-71.3$ & 94.55 & $88.5-98.0$ \\
\hline
\end{tabular}

BMI: Body mass index; HDL-C: High density lipoprotein cholesterol; LDL-C: Low density lipoprotein cholesterol; VLDL-C: Very low density lipoprotein cholesterol; CI: Confidence Interval

We further plotted the ROC curves for BMI, TC, TGs, HDL cholesterol, LDL cholesterol and VLDL cholesterol in T2DM patients with nephropathy. The significant $(\mathrm{p}<0.0001)$ area under the ROC curve was found for BMI (AUC=0.825), TC (AUC=0.724), TGs (AUC=0.725), HDL cholesterol (AUC=0.823), LDL cholesterol (AUC=0.730) and VLDL cholesterol (AUC=0.725) with corresponding cut off values of $>24.3$, $>187.2,>143.1, \leq 42.7,>119.9$ and $>37.2$, respectively with the highest sensitivity and specificity. The values of area under the ROC curve with $95 \%$ confidence interval and respective p values are depicted in table 2 . Table 3 indicates the cut off values for these parameters at highest specificity and sensitivity with respective $95 \%$ confidence intervals for T2DM patients with nephropathy and figure $2 \mathrm{a}$ to $2 \mathrm{f}$ indicates the ROC curves including $95 \%$ confidence boundaries, plotted against 100-specificity.
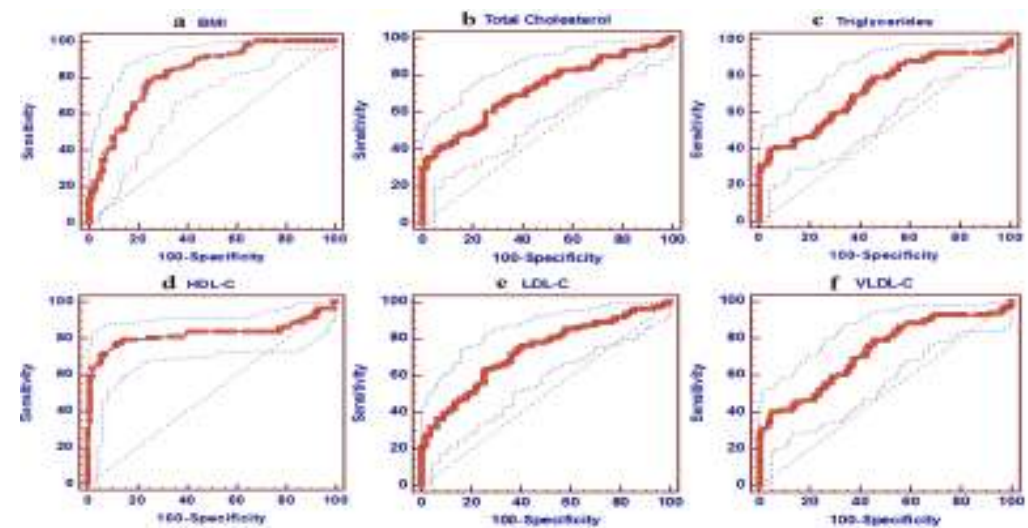

Figure 2- a to f: The ROC curves for BMI and cardiovascular markers in patients with type 2

diabetic nephropathy

\section{Discussion}

Diabetes with its characteristic hyperglycemia shows long term complications on various organs, therefore regular monitoring of organ functions for early preventive interventions of these complications is 
necessary. [12] Renal impairment is one of such common complications of diabetes mellitus. [13] Kidney damage is already present in some type 2 diabetic patients at the time of diagnosis of diabetes. [14] About one third of the diabetic patients develop microalbuminuria, nearly after few years of onset of diabetes. The overt nephropathy is developed in 50\% of microalbuminuric patients with simultaneous increase in risk of cardiovascular disease. [13] The process starts from the early stages of diabetic nephropathy to ESRD, cardiovascular diseases also progressively develop in these patients and cardiovascular diseases (CVDs) are the most important reason of the mortality in DN patients. [15]

Use of the ACR in urine samples is recommended as the preferred screening strategy for albuminuria in diagnosis of diabetic nephropathy in diabetic patients. $[15,16]$ Relation of increased urine ACR with Apo B containing lipoproteins has also been proved previously. [8] The present study attempted to find out the association of ACR with biochemical and cardiovascular markers in diabetic nephropathy patients. The positive association of ACR was found with BMI, FBG, TGs, VLDL cholesterol, Urine creatinine \& Urine microalbumin, while it is negatively associated with HDL cholesterol. Further, we calculated the cut-off values of BMI and lipid markers for the prediction of cardiovascular disease risk in diabetic nephropathy patients by means of ROC curve analysis. With significant area under the ROC curve, we found the cut off values of $>24.3$, $>187.2,>143.1, \leq 42.7,>119.9$ and $>37.2$ for BMI, total cholesterol, triglycerides, HDL cholesterol, LDL cholesterol and VLDL cholesterol, respectively.

In accordance with our results, a significant positive correlation of urine ACR was observed with plasma creatinine, and urine microalbumin in a study of Karar et al. [12] In a study population, Zhang et al. [17], found positive association of ACR with LDL cholesterol and log of LDL cholesterol/HDL cholesterol ratio in men, in females none of the lipids and lipid related ratios were associated with ACR. Sun et al. [18] reported association of ACR with the levels of TGs, HDL cholesterol, Non-HDL cholesterol/HDL cholesterol ratio, TGs/HDL cholesterol ratio, and compared to other lipids and their ratios. TGs/HDL cholesterol ratio was reported by the authors to show highest rise with increased level of ACR and odds of chronic kidney disease.

There is also a report contrasting to the results observed in the present study. Study by Bose et al. [9] found association of microalbuminuria and macroalbuminuria with $\mathrm{HbA} 1 \mathrm{c}$ levels, and no association was found for other measured lipids. Increase in HDL cholesterol was noted with decreased incidence of microalbuminuria, while no significant association of lipid markers was found with urinary ACR.

\section{Conclusion}

The ACR, calculated simply from urine albumin and urine creatinine excretion, can be utilized as an indicator of cardiovascular disease risk in type 2 diabetic nephropathy patients. The normal ranges for lipid profile of general population cannot be used to assess the risk of CVDs in type 2 diabetic nephropathy patients. Therefore, we defined the cut off points for lipid markers; these values can be used to predict the future risk of CVDs in type 2 diabetic patients with nephropathy.

\section{Acknowledgements}

The authors are thankful to BVDU Medical College and Hospital, Pune and the staff for providing the samples for present study and allowing us to use the facilities for practical work.

\section{References}

[1]. Deshpande AD, Hayes MH, Schootman M: Epidemiology of diabetes and diabetes-related complications. Phys Ther. 2008; 88(11):1254-1264

[2]. Evans TC, Capell P: Diabetic nephropathy. Clinical Diabetes, 2000; 18(1):7-16

[3]. Jalal DI, Maahs DM, Hovind P, Nakagawa T: Uric acid as a mediator of diabetic nephropathy. Semin Nephrol. 2011; 31(5):459465

[4]. Narres M, Claessen H, Droste S, Kvitkina T, Koch M, Kuss O et al:: The incidence of end-stage renal disease in the diabetic (compared to the non-diabetic) population: A Systematic Review. PLOS ONE, 2016; 11(1):e0147329

[5]. Cade WT: Diabetes-related microvascular and macrovascular diseases in the physical therapy setting. Phys Ther. 2008; 88(11):1322-1335.

[6]. Sharma V, Sharma PL: Role of different molecular pathways in the development of diabetes- induced nephropathy. J Diabetes Metab, 2013; S9:004

[7]. Pradeep Kumar Dabla: Renal function in diabetic nephropathy. World J Diabetes. 2010; 1(2):48-56

[8]. Tseng CH: Lipid abnormalities associated with urinary albumin excretion rate in Taiwanese type 2 diabetic patients. Kidney International, 2005; 67:1547-1553

[9]. Bose S, Bomback AS, Mehta NN, Chen SC, Li S, Connell AW et al and Peter A McCullough KEEP investigators: Dysglycemia but not lipids is associated with abnormal urinary albumin excretion in diabetic kidney disease: a report from the Kidney Early Evaluation Program (KEEP). Nephrology, 2012;13:104

[10]. Sung KC, Ryu S, Lee JY, Lee SH, Cheong ES, Hyun YY et al: Urine Albumin/Creatinine ratio below $30 \mathrm{mg} / \mathrm{g}$ is a predictor of incident hypertension and cardiovascular mortality. J Am Heart Assoc. 2016;5:e003245

[11]. Stern MP: Diabetes and cardiovascular disease: the "Common Soil" hypothesis. Diabetes, 1995; 44(4):369-374

[12]. Karar T, Alniwaider RAR, Fattah MA, Tamimi WA, Alanazi A, Qureshi S: Assessment of microalbuminuria and albumin creatinine ratio in patients with type 2 diabetes mellitus. J Nat Sci Biol Med. 2015; 6(1):S89-S92. 
[13]. Gross JL, De Azevedo MJ, Silveiro SP, Canani LH, Caramori ML, Zelmanovitz T: Diabetic nephropathy: diagnosis, prevention, and treatment. Diabetes Care, 2005; 28(1):164-76

[14]. Shahbazian H, Rezaii I: Diabetic kidney disease; review of the current knowledge. J Renal Inj Prev, 2013; 2(2):73-80

[15]. Bilgi M, Keser A, Katlandur H, Sahin E, Kalkan AO, Yildiz M. et al: Evaluation of the relationship between microalbuminuria and urine ischemia-modified albumin levels in patients with diabetic nephropathy. Journal of Clinical Laboratory Analysis. 2016; DOI: $10.1002 /$ jcla.22058

[16]. Momin AA, Naik PS, Bhoite GM: Albumin/creatinine ratio, as a predictor of microalbuminuria, a risk factor for nephropathy in type 2 diabetes mellitus patients. International Journal of Health Sciences \& Research, 2012; 1(2):36-40

[17]. Zhang L, Yuan Z, Chen W, Chen S, Liu X, Liang Y et al: serum lipid profiles, lipid ratios and chronic kidney disease in a Chinese population. Int. J. Environ. Res. Public Health 2014; 11:7622-7635

[18]. Sun K, Lin D, Li F, Huang C, Qi Y, Xue S. et al: Discordant associations of lipid parameters with albuminuria and chronic kidney disease: a population-based study. Lipids in Health and Disease, 2015; 14:152 